\title{
On the Comparative Performance Analysis of PAPR Reduction Techniques in OFDM System
}

\author{
Md. Ibrahim Khalil ${ }^{* 1}$ and Sabbir Ahmed ${ }^{2}$ \\ ${ }^{1}$ IPVision Canada Inc, Dhaka, Bangladesh \\ ${ }^{2}$ Dept.of Information and Communication Engineering, University of Rajshahi, \\ Rajshahi 6205, Bangladesh.
}

* Corresponding Author: khalil5614@gmail.com

\begin{abstract}
Selected Mapping (SLM) and Partial Transmit Sequence (PTS) are two very well-known Peak-to-average Power Ratio (PAPR) reduction techniques for Orthogonal Frequency Division Multiplexing (OFDM) systems. Both these schemes show good PAPR reduction capabilities. However, for any PAPR reduction technique, the nature of spectral occupancy and the associated computational complexity also need to be taken into account when the overall performance is considered. In this paper, our goal is to perform a comparative performance analysis of SLM and PTS techniques by considering these three parameters, i.e. PAPR reduction, computational overhead and spectral compactness. For this, we at first look for the optimum values in terms of number of sequences in SLM and no. of sub-blocks in PTS. And then based on this finding, we perform performance analysis. Our finding shows that, PTS outperforms SLM when compared on the parameters mentioned above. Finally, to explore the feasibility of further improvement, we apply Walsh-Hadamard Transform to PTS scheme and show that it further reduces PAPR and improves spectral compactness.
\end{abstract}

Keywords: OFDM, PAPR, CCDF, PTS, SLM, Spectral Occupancy, Walsh-Hadamard Transform.

\section{INTRODUCTION}

Orthogonal Frequency Division Multiplexing (OFDM) isa multicarriermodulation technique that is capable of achieving high data rate, goodspectral efficiency, robustness in frequency selective fading channelsand immunity to inter-symbol interference [1]. This technique is already being used in Digital Audio and video Broadcasting (DAB and DVB), LAN (IEEE802.11x) and WiMAX and so forth [1][2].

There are certain challenges in implementing OFDM also.In particular, the issue of high Peak to Average Power Ratio (PAPR) of the transmitted signal[3] needs special attention. High PAPR may drive transmitter high power amplifier (HPA) into its saturation region resulting in non-linear amplification. This non-linear amplification eventually results in severe bit error rate (BER) degradation in the receiver. One solution to this problem is to operate HPAs with high-back offs to compensate for large peaks present in the signal. But this yields in very poor power efficiency. Since, wireless handheld terminals are by nature power constrained, it is imperative that reduction of PAPR should get the focus rather than taking compensating measures by sacrificing power efficiency.

Because of the reasons mentioned above, PAPR reduction problem has drawn significant research interests and as aresult quite a significant number of methods of PAPR reduction 
can be found in literature. Amongst themsignal clipping, selected mapping (SLM) and partial transmit sequence (PTS) are considered by many as the fundamental PAPR reduction methods. In signal clipping method, the peaks in the signal above a certain prescribed level are clipped. This is a very simple technique. But clipping any signal causes in-band distortion and out of band radiation; both resulting in higher BER. On the other hand, SLM and PTS methods are classified asdistortion-less phase control schemes. In SLM, multiple signals are generated by multiplying the information bearing signalwith pre-decided phase sequences. And then among these multiple generated signals, signalwiththe lowest PAPR is selected for transmission [4]. Whereas in PTS, the lowest PAPR signal is constructedby optimally phase combining multiple signal sub-blocks [5].

Both SLM and PTS are flexible methods and have good PAPR reduction capability. But they impose added computational overhead on the system. In this respect, the number of different phase sequences (U) in SLM and the number of sub-blocks (V) in PTS are the two parameters that are of importance. While higher values of $\mathrm{U}$ and $\mathrm{V}$ increase the probability of achieving better PAPR reduction, on the downside they also increase the computational complexity. Again the spectral occupancy for different values of $\mathrm{U}$ and $\mathrm{V}$ may be different since they essentially represent different signals. The issue of retaining spectral compactness is also very important. Since trading spectral efficiency for PAPR reduction may not be acceptable as it refers to nothing but losing one of the fundamental advantages of OFDM i.e. high spectral efficiency.

In light of all the above mentioned observations, we were motivated to investigate the comparative

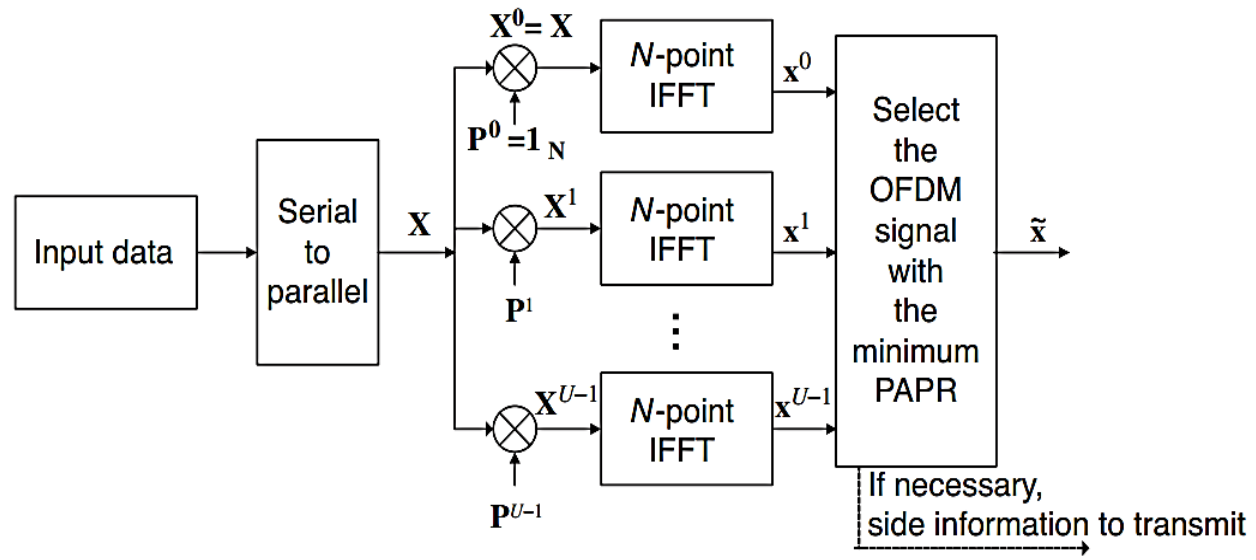

Fig. 1: Block diagram of selective mapping (SLM) technique for PAPR reduction.

performance of SLM and PTS from three different perspectives i.e. PAPR reduction ability, computational complexity and finally spectral efficiency. In particular, after determining acceptable values of $U$ and $V$ with reasonable PAPR reduction, we compare SLM and PTS on their spectral performance and recommend PTS over SLM. Hadamard transform may reduce PAPR of OFDM signal. 


\section{PEAK TOAVERAGE POWER RATIO (PAPR)}

The Peak to Average Power Ratio (PAPR) of OFDM is defined as the ratio between the maximum instantaneous powerand the average power. The PAPR (in $\mathrm{dB}$ ) of the transmitted OFDM signal can be written in mathematical expression as:

$$
\operatorname{PAPR}(\mathrm{dB})=10 \log _{10} \frac{\max \left[|\mathrm{X}[\mathrm{n}]|^{2}\right]}{\mathrm{E}\left[|\mathrm{X}[\mathrm{n}]|^{2}\right]}, 0 \leq \mathrm{t} \leq \mathrm{T}
$$

Where $\mathrm{X}[\mathrm{n}]$ denotes an OFDM signal after IFFT and E[.] denotes expectation. The complex time domain OFDM signal for $\mathrm{N}$ subcarriers can be represented as

$$
X[n]=\frac{1}{\sqrt{N}} \sum_{k=0}^{N-1} x[k] e^{\frac{j 2 \pi n k}{N}}, 0 \leq n \leq N-1
$$

However in practice, it is preferred to take the probability of PAPR exceeding a threshold as the measurement index to represent the distribution of PAPR. This is described as Complementary Cumulative Distribution Function (CCDF) and is mathematically expressed as:

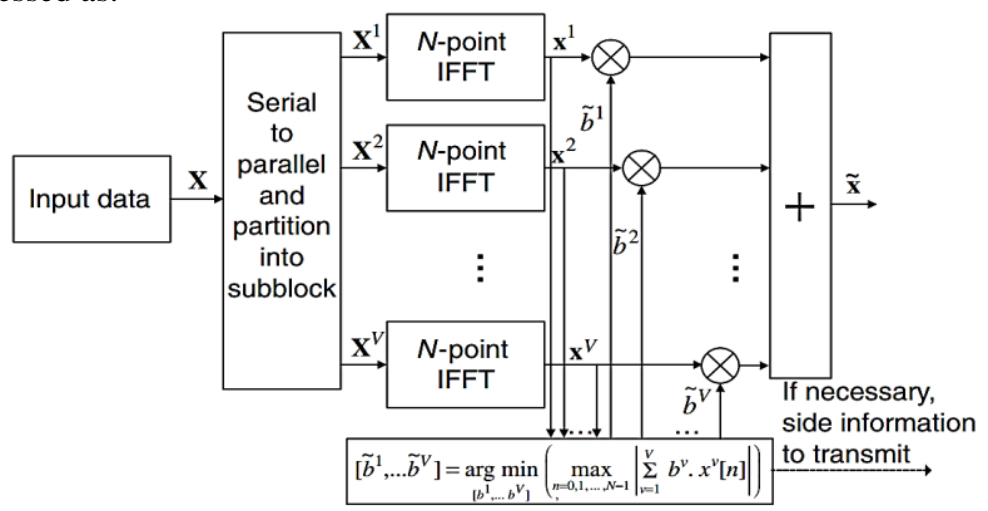

Fig. 2: Block diagram of partial transmit sequence (PTS) technique for PAPR reduction.

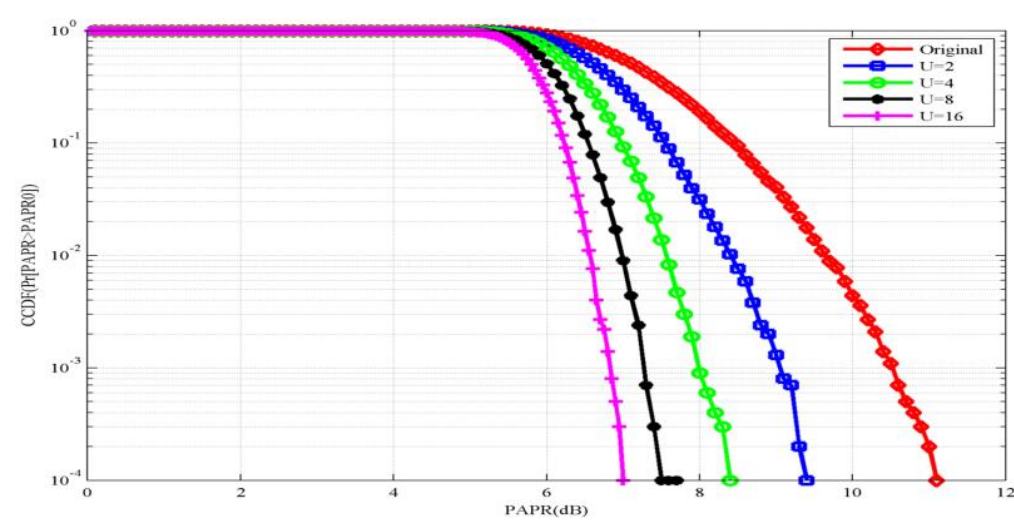

Fig. 3: Performance of SLM PAPR reduction technique for different values of $U$ where $N=64$

$$
\operatorname{Pr}(\operatorname{PAPR}>z)=1-(1-\exp (-\mathrm{z}))^{\mathrm{N}}
$$

Where $\mathrm{z}$ is the threshold value and $\mathrm{N}$ is the number of sub-carrier. 


\section{PAPR REDUCTION TECHNIQUES}

There are many different techniques that have beenproposed to provide a solution to the problem of high PAPRof OFDM system. Among these techniques, this paper is focusedon Selective Mapping (SLM) and Partial Transmit Sequences(PTS) [6]. These are discussed below.

\section{A. SELECTED MAPPING (SLM)}

The main objective of this technique is to generate a set ofdata blocks at the transmitter end which representthe original information and then to choose the most favorable block among them for transmission. Fig. 1 shows the block diagram of selective mapping (SLM) technique for PAPR reduction.

Here, the input data block $\mathrm{X}=[\mathrm{X}[0], \mathrm{X}[1], \ldots, \mathrm{X}[\mathrm{N}-1]]$ is multiplied with $\mathrm{U}$ different phase sequences $\mathrm{P}^{\mathrm{u}}=\left[\mathrm{P}_{0}^{\mathrm{U}}, \mathrm{P}_{1}^{\mathrm{U}}, \ldots, \mathrm{P}_{\mathrm{N}-1}^{\mathrm{U}}\right]^{\mathrm{T}}$ where $\mathrm{P}_{\mathrm{v}}^{\mathrm{u}}=\mathrm{e}^{\mathrm{j} \varphi_{\mathrm{v}}^{\mathrm{u}}}$ and $\varphi_{\mathrm{v}}^{\mathrm{u}} \in[0,2 \pi]$ for $\mathrm{v}=$ $0,1, \ldots, \mathrm{N}-1$ and $\mathrm{u}=1,2, \ldots, \mathrm{U}$, whichproduce $\mathrm{a}$ modified data block $\mathrm{X}^{\mathrm{u}}=\left[\mathrm{X}^{\mathrm{u}}[1], \mathrm{X}^{\mathrm{u}}[2], \ldots, \mathrm{X}^{\mathrm{u}}[\mathrm{N}-1]\right]^{\mathrm{T}}$. IFFT of $\mathrm{U}$ Independentsequences $\left\{\mathrm{X}^{\mathrm{u}}[\mathrm{v}]\right\}$ are taken to produce the sequences $\mathrm{x}^{\mathrm{u}}=\left[\mathrm{x}^{\mathrm{u}}[0], \mathrm{x}^{\mathrm{u}}[1], \ldots, \mathrm{x}^{\mathrm{u}}[\mathrm{N}-1]\right]^{\mathrm{T}}$ among which the one $\widetilde{\mathrm{x}}=$ $\mathrm{x}^{\widetilde{\mathrm{u}}}$ with the lowest PAPR is selected for transmission, as shown as

$$
\tilde{u}=\underset{u=1,2, \ldots, U}{\operatorname{argmin}}\left(\left.\begin{array}{c}
\max \\
n=0,1, \ldots, N-1
\end{array}\right|^{u}[n] \mid\right)
$$

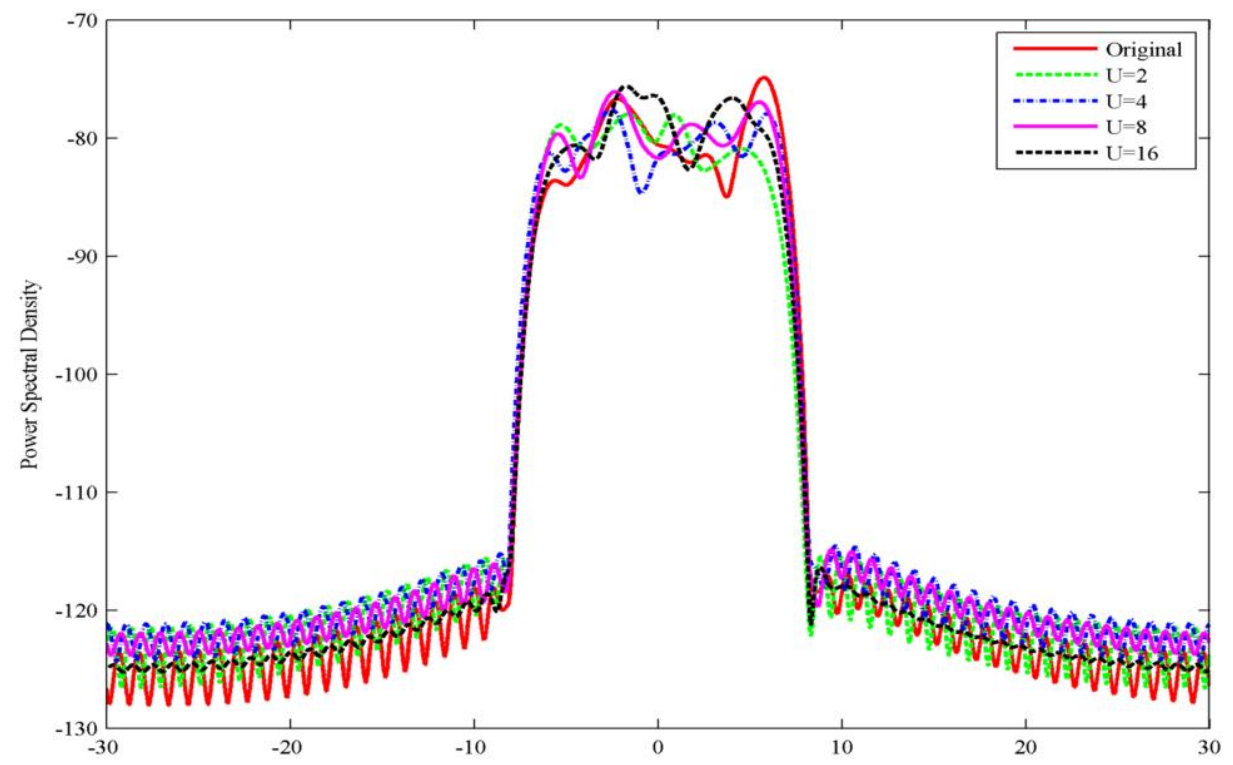

Fig. 4: Spectral Occupancy or Out of Band radiation of SLM technique different value of $U$ with $N=64$

In order for the receiver to be able to recover the original data block, the information (index u) about the selected phase sequence $P^{u}$ should be transmitted as side information. PAPR is decrease when the number of sequence increases. 
TABLE 1: SiMULATION PARAMETERS

\begin{tabular}{|c|c|}
\hline Parameter & Value/Description \\
\hline Number of OFDM symbol & 10000 \\
\hline Number of Subcarrier & 64 \\
\hline Number of data per subcarrier & 52 \\
\hline Modulation & $4 Q A M$ \\
\hline Channel & AWGN \\
\hline Over-sampling factor(L) & 4 \\
\hline Number of Sub-block(V) & $2,3,4$ \\
\hline Number of sequence(U) & $2,4,8,16$ \\
\hline
\end{tabular}

B. PARTIAL TRANSMIT SEQUENCE (PTS)

In the partial transmit sequence (PTS) technique an input data block of $\mathrm{N}$ symbols is partitioned into $\mathrm{V}$ disjoint sub-blocks as follows:

$$
\mathrm{X}=\left[\mathrm{X}^{0}, \mathrm{X}^{1}, \mathrm{X}^{2}, \ldots, \mathrm{X}^{\mathrm{V}-1}\right]^{\mathrm{T}}(5)
$$

Where $X^{i}$ are the sub-blocks that are consecutively located and also are of equal size. Unlike the SLM technique in which scrambling is applied to all subcarriers, scrambling (rotating its phase independently) is applied to each sub-block in the PTS technique (see Fig. 2). Then each partitioned sub-block is multiplied by a corresponding complex phase factor $\mathrm{b}^{\mathrm{v}}=\mathrm{e}^{\mathrm{j} \phi \mathrm{v}}, \mathrm{v}=1,2, \ldots, \mathrm{V}$, subsequently taking its IFFT to yield

$$
\mathrm{x}=\operatorname{IFFT}\left\{\sum_{\mathrm{v}=1}^{\mathrm{V}} \mathrm{b}^{\mathrm{v}} \mathrm{X}^{\mathrm{v}}\right\}=\sum_{\mathrm{v}=1}^{\mathrm{v}} \mathrm{b}^{\mathrm{v}} \cdot \operatorname{IFFT}\left\{X^{\mathrm{v}}\right\}=\sum_{\mathrm{v}=1}^{\mathrm{V}} \mathrm{b}^{\mathrm{v}} \mathrm{X}^{\mathrm{v}}
$$

Where $\left\{\mathrm{X}^{\mathrm{v}}\right\}$ is referred to as a partial transmit sequence (PTS).

The phase vector is chosen so that the PAPR can be minimized, which is shown as

$$
\left[\tilde{\mathrm{b}}^{1}, \ldots \tilde{\mathrm{b}}^{\mathrm{v}}\right]=\underset{\left[\mathrm{b}^{1}, \ldots, \mathrm{b}^{\mathrm{V}}\right]}{\operatorname{argmin}}\left(\mathrm{n}=0,1, \ldots, \mathrm{N}-1\left|\sum_{\mathrm{v}-1}^{\mathrm{V}} \mathrm{b}^{\mathrm{v}} \mathrm{x}^{\mathrm{v}}[\mathrm{n}]\right|\right)
$$

Then, the corresponding time-domain signal with the lowest PAPR vector can be expressed as:

$$
\tilde{\mathrm{x}}=\sum_{\mathrm{v}=1}^{\mathrm{V}} \tilde{\mathrm{b}}^{\mathrm{v}} \mathrm{x}^{\mathrm{v}}
$$

The PTS technique requires V IFFT operations for each data block and $\log _{2} \mathrm{~W}^{\mathrm{V}}$ bits of side information [7].

\section{HADAMARD TRANSFORM}

The idea to use the Hadamard Transform is to reduce the autocorrelation of the input sequence to reduce the peak to average power problem and it requires no side information to be transmitted to the receiver. In the section, we briefly review Hadamard Transform. We assume $\mathrm{H}$ is the Hadamard Transform matrix of $\mathrm{N}$ orders, and Hadamard matrix is standard orthogonal matrix. Every element of Hadamard matrix only is 1 or -1 . The Hadamard matrix of 2 orders is stated by

$$
\mathrm{H} 2=\frac{1}{\sqrt{2}}\left[\begin{array}{cc}
1 & 1 \\
1 & -1
\end{array}\right]
$$

Hadamard matrix of order $\mathrm{N}$ may be constructed by 


$$
\mathrm{H} 2=\frac{1}{\sqrt{2 \mathrm{~N}}}\left[\begin{array}{cc}
\mathrm{Hn} & \mathrm{Hn} \\
\mathrm{Hn} & -\mathrm{Hn}
\end{array}\right]
$$

TABLE 2: COMPUTATIONAL COMPLEXITY OF SLM TECHNIQUE

\begin{tabular}{|c|c|}
\hline Sequence, $\mathbf{U}$ & Computational Complexity \\
\hline 2 & 768 \\
\hline 4 & 1536 \\
\hline 8 & 3072 \\
\hline 16 & 6144 \\
\hline
\end{tabular}

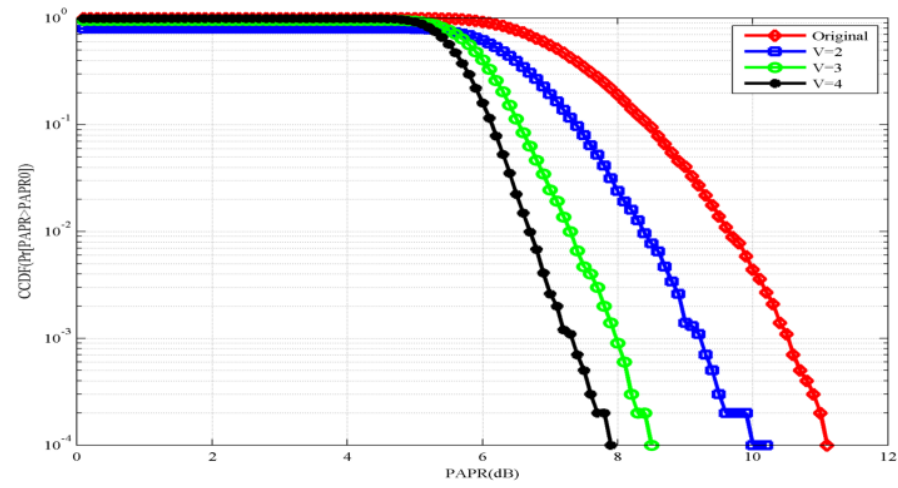

Fig. 5: Performance of PTS technique for different number of sub-blocks V with $\mathrm{N}=64$

After the sequence $\mathrm{X}=[\mathrm{X} 1, \mathrm{X} 2 \ldots \mathrm{Xn}]$ is transformed by Hadamard matrix of $\mathrm{N}$ order, the new sequence is

$$
\mathrm{Y}=\mathrm{HX}
$$

The coming input data stream is firstly transform by the Hadamard Transform then the transformed data stream is applied as input to IFFT signal processing unit.

\section{COMPUTATIONALCOMPLEXITY}

Computational complexity depends of number of IFFT operations in OFDM signal. A total V. $\mathrm{W}^{\mathrm{V}}$ number of IFFToperations performed in PTS technique, where $\mathrm{V}$ is the number of sub-block and $\mathrm{W}$ is the possible variations of the phase. In SLM, every time when SLM technique is applied, it requires calculating the U group IFFTs at the transmitter compared to only one on ordinary OFDM systemand its $\mathrm{U}$ of $\mathrm{N}$ pointsIFFTs operation needsn $n_{\text {mul }}=U \cdot \frac{\mathrm{N}}{2} \log _{2} \mathrm{~N}$ complex multiplication and $\mathrm{n}_{\text {add }}=\mathrm{U} \cdot \frac{\mathrm{N}}{2} \cdot \log _{2} \mathrm{~N}$ addition separately. These problems usually pose high difficulties on real OFDM implementation; therefore, it is required to reduce the computational complexity [6].

\section{SIMULATION RESULTS AND DISCUSSION}

For comparative performance analysis, we performed simulation based on the model shown in Figs. 1 and 2. We considered QAM modulation and investigated scenarios for sequence $\mathrm{U}=2,4,8,16$ and sub-block $\mathrm{V}=2,3$, 4. All the pertinent simulation parameters are listed in Table I. 


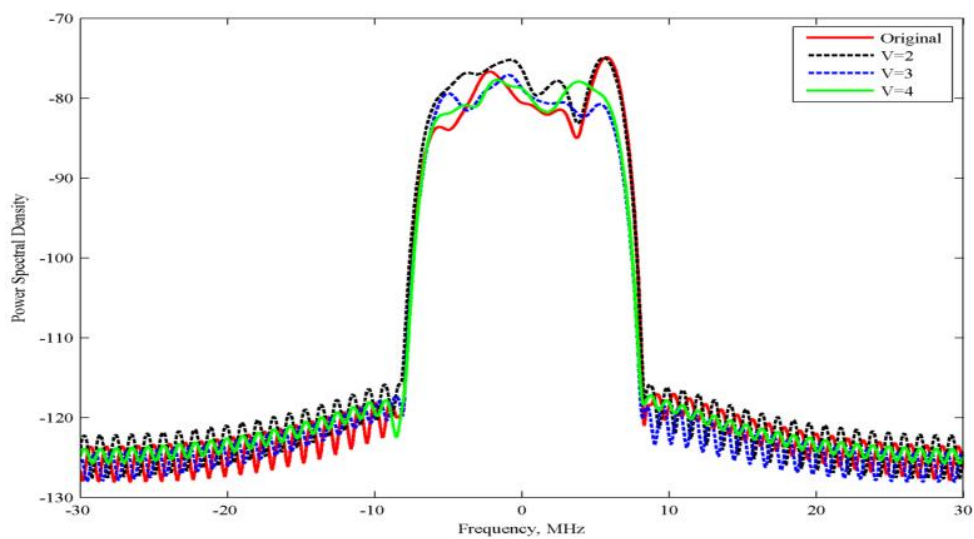

Fig. 6: Spectral Occupancy or Out of Band radiation of PTS technique with N=64

TABLE 3: COMPUTATIONAL COMPLEXITY OF PTS

\begin{tabular}{|c|c|}
\hline Number of Sub-block & Computational Complexity \\
\hline 2 & 512 \\
\hline 3 & 768 \\
\hline 4 & 1024 \\
\hline
\end{tabular}

Figure 3 shows the performance of SLM PAPR reduction technique for different values of sequence $U$ with subcarrier size $N=64$. From this figure it is observed that SLM technique reduces PAPR significantly compared to the original OFDM signal. When the number of sequence $U$ is increased, the PAPR decreases. But with increasing $U$, the computational complexity also increases. However, it is difficult to achieve linear growth of PAPR reduction performance with further improvement of $U$ (like $U>=8$ ).

Figure 4shows the spectral occupancy or out of band radiation performance of SLM technique for different value of sequence $U$ with subcarriersize $N=64$. Here, it is observed that, the spectral occupancy performance of SLM method improves for the value of U (like $\mathrm{U}>=8$ ).

From the above discussion, we understand that if computational complexity is not taken intoconsideration, higher values of $U$ e.g. $U=16$ serve the purpose. But $U=16$ has high computational complexity. In addition, with $U=16$ we get PAPR reduction is not significantly high when compared to the high computational complexity. Hence we preferred lower value of $U$ i.e. $U<=8$, so as to avoid introducing too much computational complexity.

Figure 5 shows the performance of PTS technique for different number of sub-blocks V with subcarrier size $\mathrm{N}=64$. From the Fig. 5, it can be observed that increasing sub-block number $\mathrm{V}$ leads to the improvement of PAPR reduction performance. Sub-block, V=4 shows better PAPR performance than $\mathrm{V}=2$ and 3.

Figure 6 shows the Spectral Occupancy performance of PTS technique for different value of sub-block with subcarrier size $\mathrm{N}=64$. From Fig. 6, it can be observed that PTS has significant out of band radiation improvement for sub block $V=4$ with compare to original OFDM signal. 
From the above observation we can decide that in practical applications, it is preferred to take sub block number $\mathrm{V}=4$ which gives better PAPR and spectral occupancy performance.

Figure 7 shows the performance of PTS and SLM PAPR reduction technique for same values of sequence $U$ and sub-block $V$ as 4 with subcarrier size $N=64$. From Fig. 7 it can beobserved that PTS method has significant PAPR reduction with compare to original OFDM signal. When both $\mathrm{U}$ and $\mathrm{V}$ are same, PTS technique give better performance than SLM technique. For the both same number of $U$ and V=4 PTS techniques gives $0.84 \mathrm{~dB}$ PAPR reduction with compare to SLM.

Figure 8 shows the spectral occupancy or out of band radiation performance of PTS and SLM PAPR reduction technique for same value of sequence $U$ and sub-block $V$ as 4 with number of subcarrier $\mathrm{N}=64$. From Fig. 8, it can be observed that PTS method has significant out-of-band radiation reduction compared to the original OFDM signal. SLM method has more out of band radiation than original OFDM signal. An improved $3.8 \mathrm{~dB}$ out of band radiation improved in PTS with compared to original OFDM signal.

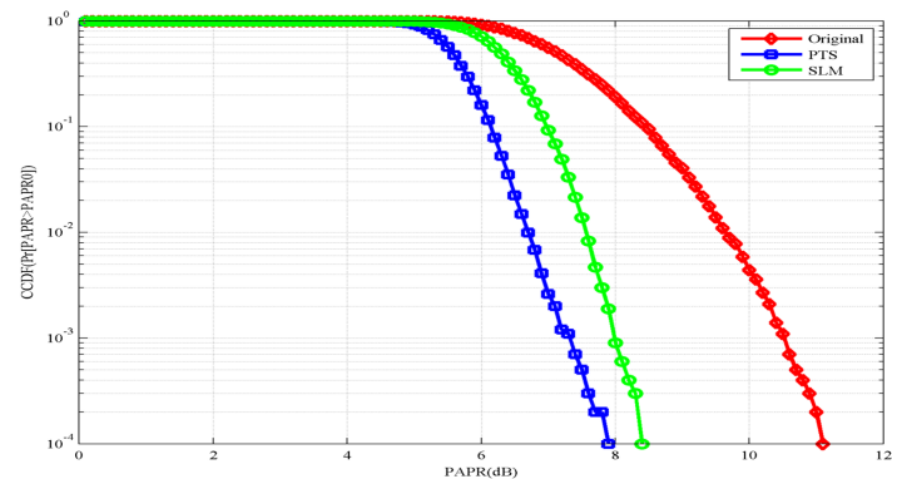

Fig. 7: Comparative PAPR for PTS and SLM PAPR reduction technique for $U=4$ and $V=4$ with $N=64$

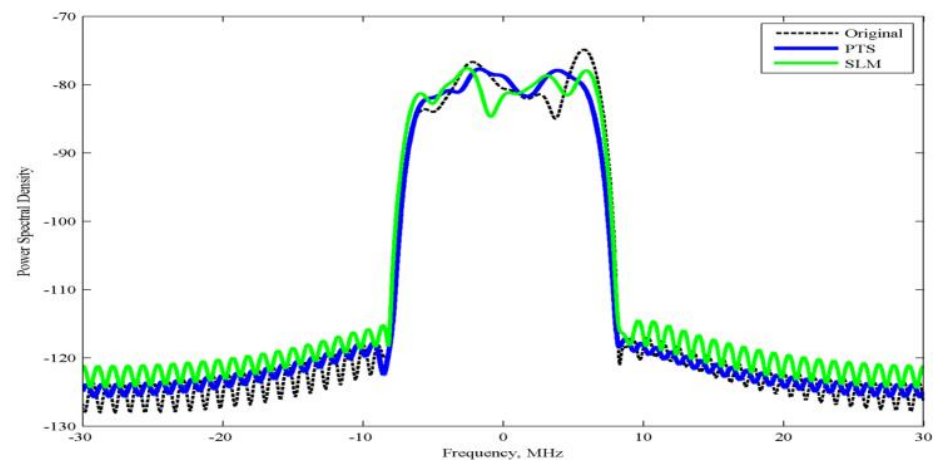

Fig. 8: Spectral Occupancy or Out of Band radiation of PTS and SLM technique for U=4 and V=4 with N=64

From the above discussion of PTS and SLM PAPR reduction technique, it can be decide that with same value of U and V PTS method has better performance. It is preferred to use PTS PAPR reduction technique for practical application. 
Figure 9 shows the performance of PTS and PTS with Walsh Hadamard Transform PAPR reduction technique for same value of sub-block, $V=4$ with subcarrier size $\mathrm{N}=64$.From Fig. 9 it can be observed that PTS with Walsh Hadamard Transform has significant PAPR reduction with compare to original OFDM signal and PTS implemented OFDM signal.For same value of sub-block, V= 4 PTS with Walsh Hadamard Transform give better performance than PTS technique.

Figure 10 shows the spectral occupancy or out of band radiation performance of PTS and PTS with Walsh Hadamard Transform PAPR reduction technique for same value of subblock V as 4 with number of subcarrier $\mathrm{N}=64$. From Figure 10 it can be observed that PTS with Walsh Hadamard Transform shows significant out of band radiation reduction with compared to original OFDM signal and PTS method implemented OFDM signal.

\section{CONCLUSION}

In this paper, we have investigated the result obtained from applying PAPR reduction technique for different value of sequence number U in SLM and sub-block number V in PTS. The performance parameters are PAPR, computational complexity and spectral occupancy. In SLM PAPR reduction technique of OFDM system, it has been observed that if computational complexity is considered, SLM technique shows better performance for

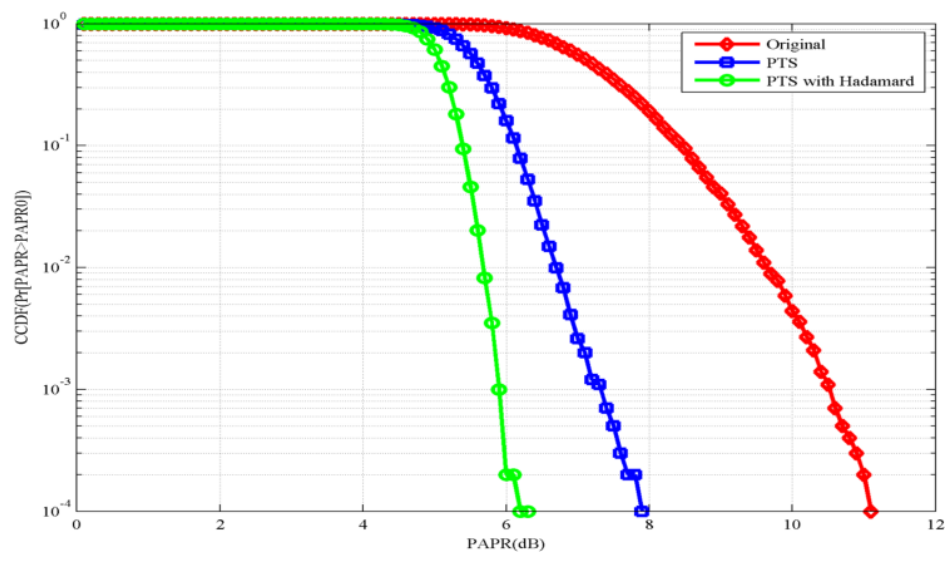

Fig. 9: Comparative PAPR for PTS and PTS with Hadamard Transform PAPR reduction techniquefor $\mathrm{V}=4$ with $\mathrm{N}=64$

sequence $U<=8$. If computational complexity is not considered, SLM technique with sequence $\mathrm{U}=16$ shows better performance. In PTS PAPR reduction technique of OFDM system, it has been observed that, PTS PAPR reduction technique shows better performance for sub- block number $\mathrm{V}=4$. Computational complexity is taken into consideration. With knowing that PTS method shows better performance for $\mathrm{V}=4$ and SLM method for $\mathrm{U}<=8$, we compare PTS and SLM method. PTS method shows better PAPR, computational complexity and spectral occupancy or out of band radiation performance than SLM. PTS method can be considered because of better spectral compactness or lower out of band radiation and less computational complexity 


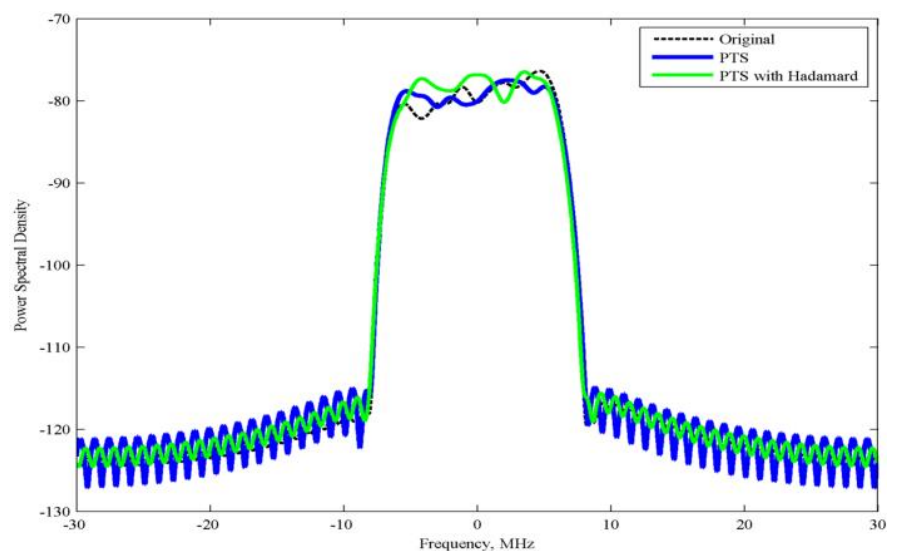

Fig. 10: Spectral Occupancy or Out of Band radiation of PTS and PTS with Hadamard Transform PAPR reduction technique with $\mathrm{N}=64$

Therefore, PTS method is the best between PTS and SLM. However, the transmitter and receiver complexity is very high. Thus in practical applications, a trade-off needs to be made between good performance and auxiliary information. From the above made discussion, SLM technique is more suitable if system can tolerate more redundant information; otherwise, PTS technique is more acceptable when complexity becomes the first considering factor. In comparison of Walsh-Hadamard Transform with PTS PAPR reduction technique and PTS PAPR reduction, it has been observed that Walsh-Hadamard Transform with PTS PAPR reduction technique shows better performance in PAPR reduction and spectral occupancy or out ofbandradiation.

\section{References}

[1] L. K. Gupta, B. Kumar,A. mishra,A. Kumar, "PAPR REDUCTION USING MODIFIED SELECTIVE MAPPING TECHNIQUE”, International Journal of Advanced Technology \& Engineering Research (IJATER), Vol. 3, Issue 3,pp. 130-133, May 2013.

[2] P. K. Sharma, R. K. Nagaria, "An SLM based PAPR Reduction Method using New VolterraPredistorter Model in the OFDM System", WSEAS TRANSACTIONS on COMMUNICATIONS, Vol. 11, Issue 10, pp. 367-374, October 2012.

[3] Mr. J. Raj, Mr. M.Malleswaran, "DCT Based Modified SLM Technique for PAPR Reduction in OFDM Transmission", International Journal of Scientific \& Engineering Research, Vol. 3, Issue 6, June-2012.

[4] R. W. Bauml, R. F. H. Fischer and J. B. Huber, "Reducing the peak-to-average power ratio of multicarrier modulation by selected mapping," Electronics Letters, vol. 32, no. 22, pp. 2056-2057, October 1996.

[5] L. J.Ciminiand N. R. Sollenberger, "Peak-to-average power ratio reduction of an OFDM signal using partial transmit sequences," IEEE Communications Letters, vol.4, no. 3, pp. 86-88, March 2000. 
[6] M. Singh, V. Kumar, "Investigation of PAPR Reduction in MIMO-OFDM System using PTS and SLM Techniques", International Journal of Science and Research (IJSR), Vol. 3 Issue 3, pp. 524- 529, March 2014.

[7] Y. S. Cho, J. Kim, W. Y. Yang, C. G. Kang, MIMO-OFDM Wireless Communications with MATLAB, John Wiley \& Sons (Asia) Pte Ltd, 2010. 